OPEN ACCESS

Edited by:

Yong Su,

Nanjing Agricultural University, China

Reviewed by:

Jianping Wang,

Sichuan Agricultural University, China

Ruizhi Hu,

Hunan Agricultural University, China

*Correspondence:

Bao Yi

yibao@caas.cn

Specialty section:

This article was submitted to

Nutrition and Microbes,

a section of the journal

Frontiers in Nutrition

Received: 22 December 2020 Accepted: 03 March 2021

Published: 06 April 2021

Citation:

Wang $M$, Zhang S, Zhong $R$, Wan F,

Chen L, Liu L, Yi B and Zhang $H$

(2021) Olive Fruit Extracts Supplement

Improve Antioxidant Capacity via

Altering Colonic Microbiota

Composition in Mice.

Front. Nutr. 8:645099.

doi: 10.3389/fnut.2021.645099

\section{Olive Fruit Extracts Supplement Improve Antioxidant Capacity via Altering Colonic Microbiota Composition in Mice}

\author{
Mengyu Wang ${ }^{1}$, Shunfen Zhang ${ }^{1}$, Ruqing Zhong ${ }^{1}$, Fan Wan ${ }^{1,2}$, Liang Chen ${ }^{1}$, Lei Liu ${ }^{1}$, \\ Bao $\mathrm{Yi}^{{ }^{*}}$ and Hongfu Zhang ${ }^{1}$ \\ 'State Key Laboratory of Animal Nutrition, Institute of Animal Science, Chinese Academy of Agricultural Sciences, Beijing, \\ China, ${ }^{2}$ College of Pastoral Agriculture Science and Technology, Lanzhou University, Lanzhou, China
}

Oxidative stress, one of the most common biological dysfunctions, is usually associated with pathological conditions and multiple diseases in humans and animals. Chinese olive fruit (Canarium album L.) extracts (OE) are natural plant extracts rich in polyphenols (such as hydroxytyrosol, HT) and with antioxidant, anti-hyperlipidemia, and anti-inflammatory potentials. This study was conducted to investigate the antioxidant capacity of OE supplementation and its related molecular mechanism in mice. Mice $(25.46 \pm 1.65 \mathrm{~g})$ were treated with $100 \mathrm{mg} / \mathrm{kg}$ body weight (BW) OE or saline solution for 4 weeks, and then the antioxidant and anti-inflammatory capacities of mice were examined. The results showed that OE supplement significantly increased the serum antioxidative enzyme activities of total antioxidant activity (T-AOC), superoxide dismutase (SOD), glutathione peroxidase (GSH-Px), and catalase and decreased the serum malondialdehyde (MDA) level, indicating that OE treatment enhanced the antioxidant capacity in mice. qPCR results showed that the transcriptional expression of antioxidant SOD1, CAT, Gpx1, and Gpx2 were significantly down-regulated in the small intestine (jejunum and ileum) after OE administration. Meanwhile, OE treatment significantly decreased the T-AOC and increased the MDA level in the small intestine. Furthermore, OE administration dramatically reduced the mRNA expression of pro-inflammatory cytokines (TNF- $\alpha$ and $\mathrm{IL}-1 \beta$ ), which confirmed its antioxidant and anti-inflammatory capacities with OE administration. Using amplicon sequencing technology, 16S rRNA sequencing results showed that OE supplement significantly increased the colonic Firmicutes/Bacteroidetes ratio, which also had a negative correlation with the serum MDA level and positively correlated with serum GSH-Px activity through Pearson correlation analysis. Besides that, Alloprevotella was negatively correlated with serum T-AOC. Colidextribacter was positively correlated with serum MDA and negatively correlated with serum T-AOC, SOD, and GSH-Px levels. In summary, this study showed that treatment with $100 \mathrm{mg} / \mathrm{kg}$ BW polyphenol-rich OE could alter colonic microbiota community, which was strongly associated with improved antioxidant capacity in mice.

Keywords: olive extracts, antioxidant capacity, gut microbiota, oxidative stress, anti-inflammatory capacity, hydroxytyrosol 


\section{INTRODUCTION}

Oxidative stress is regarded as a result of the imbalance of oxidants and antioxidants, which can cause damage to important cellular macromolecules, such as DNA, lipid, and protein, and, in turn, lead to toxicity, chronic inflammation, and diseases, acting as a serious threat to animal and human health (1-3). In animal husbandry, oxidative stress is commonly considered to be associated with various pathological conditions and can severely damage productivity and livestock product quality and even lead to death $(4,5)$. Similarly, oxidative stress is also an important factor for the progression of human diseases and body disorders, including metabolic diseases and inflammationrelated diseases, such as inflammatory bowel disease and diabetes (6-8). Moreover, the overproduction of reactive oxygen species and reactive nitrogen species during oxidative stress can cause inflammatory responses by activating the related signal transduction pathways $(9,10)$.

Polyphenols are natural compounds present in plants with numerous biological activities, which have been proposed to be useful as adjuvant therapy for their potential antioxidant effect, associated with the anti-inflammatory activity (11). Olive extracts, as one of the important natural plant extracts, have been extensively explored for their potential antioxidant properties $(12,13)$. The main bioactive component of olive extracts are polyphenols, which are thought to be responsible for their wide range of biological activities. Increasing evidence has indicated that olive extracts rich in polyphenolic compounds have powerful antioxidant and anti-inflammatory effects in mammalian cells, rats, and humans (14-17). Administration of olive oils high in phenolic compounds decreased malondialdehyde (MDA) levels in urine and increased plasma glutathione peroxidase (GSH-Px) activity in a dose-dependent manner in men (18). Olive leaf extract could enhance antioxidation capacity in the liver of aged mice by inducing a decrease in the MDA level and an increase in glutathione (GSH) level (19). Olive pomace extracts supplement followed with increased total antioxidant activity (T-AOC) was shown in the study of A. De Bruno et al. (20). In addition, some studies have shown that olive extracts have anti-bacterial and anti-inflammation effects (21-23). It is well-established that these beneficial health properties of olive extracts are related to one of the polyphenolic compounds named hydroxytyrosol (HT) (2426). Studies in mammalian cells have demonstrated that HT can exert potential effects against oxidative stress and inflammation $(24,27)$. Further mechanism research showed that HT alleviated oxidative stress by decreasing the production of oxygen species (24). Thus, olive extracts enriched with various polyphenols (especially HT) may be an effective prevention against disorders related to oxidative stress.

Concerning the metabolism of olive extracts, particularly the olive bioactive component polyphenols, growing evidence has demonstrated that only small amounts of ingested polyphenols can be absorbed in the small intestine and enter the systemic circulation $(28,29)$. Most remaining polyphenols reach into the large intestine, where they can be metabolized by gut microbiota $(28,29)$. The colonic microbiota, therefore, plays a key role in the metabolism of polyphenols. A study showed that olive administration could alleviate hypercholesterolemia by reducing the relative abundance of Lactobacilli and Ruminococcus in the human gut microbiota (30). Extra virgin olive oil supplementation increased the gut microbiota diversity and decreased the relative abundance of Firmicutes in mice (31). In addition, another study showed that olive leaf extract can counteract the ecological disorders associated with obesity by altering the colonic microbial community in mice (32). These studies have suggested that olive oil and olive leaf phenolic compounds can induce changes in gut microbial composition and alter its metabolism in mice and humans with metabolic diseases.

However, whether oral administration of Chinese olive fruit (Canarium album L.) extracts (OE) could improve colonic microbiota and whether colonic microbiota is a remarkable mechanism further involved in antioxidant and anti-inflammatory effects of $\mathrm{OE}$ remain to be elucidated. We here, therefore, investigated the effects of $\mathrm{OE}$ on the levels of antioxidant indicators, the expressions of antioxidant enzymes and inflammatory cytokines in the intestine, and the colonic microbiota composition to explore its underlying molecular mechanism.

\section{MATERIALS AND METHODS}

\section{Reagents, Mice, and Ethics}

The OE was purchased from Shanghai Huahan Biotechnology Co., Ltd. (Shanghai, China), and it was composed of $10 \mathrm{wt} \%$ HT as checked by high-performance liquid chromatography. Specifically, an Acquity UPLC BEH C18 $(1.7 \mu \mathrm{m}, 2.1 \times 50 \mathrm{~mm})$ column was used. The binary mobile phase consists of two different formic acid solutions running in a linear gradient, and detection is carried out with UV-vis at $278 \mathrm{~nm}$. Quantification was performed by the external standard method with tyrosol and HT reference standards. Then, the concentrations of these compounds were calculated using the response factor of HT reference standard. Three-week-old female ICR mice were purchased from Peking University Health Science Center (Beijing, China). The mice were maintained in a 12-h light/dark cycle, with free access to diet and water. All procedures used in this experiment were approved by the Experimental Animal Welfare and Ethical Committee of the Institute of Animal Science, Chinese Academy of Agricultural Sciences (no. IAS2020-86).

\section{Mice Experiment and Sampling}

After 1 -week acclimatization, the mice $(25.46 \pm 1.65 \mathrm{~g})$ were divided into two groups ( $n=12$ per group). The OE supplement (OE) group was treated with $100 \mathrm{mg} / \mathrm{kg}$ body weight $\mathrm{OE}$ (prepared fresh in distilled water before gavage), and the control (Con) mice received the same volume of distilled water every day via oral gavage. Body weight and feed intake of the mice were measured weekly. At the end of day 28, blood samples were collected by orbital blooding, and then the mice were killed by cervical dislocation. The jejunum and ileum tissues were quickly removed and frozen in liquid nitrogen for further analysis. For histopathology examinations, part of the jejunum and ileum 
were cut and fixed in $4 \%$ paraformaldehyde. Colonic digesta were collected for $16 \mathrm{~S}$ rRNA sequencing and short-chain fatty acid analysis.

\section{Serum Oxidant and Antioxidant Marker Analyses}

Serum was obtained by centrifugation at $1,000 \mathrm{~g}$ for $15 \mathrm{~min}$ under $4^{\circ} \mathrm{C}$ and stored in aliquots at $-80^{\circ} \mathrm{C}$. The activities of total antioxidant capacity (T-AOC), glutathione peroxidase (GSH-Px), catalase (CAT), and superoxide dismutase (SOD) and the level of malondialdehyde (MDA) and inflammatory cytokines (TNF$\alpha$, IL-1 $\beta$, IL-6, and IFN- $\gamma$ ) were measured with corresponding assay kits (Nanjing Jiancheng Bioengineering Institute, Nanjing, China) following the manufacturers' instructions.

\section{Intestinal Morphology Examination}

Proximal jejunum and distal ileum sections were used for histologic examination. They were fixed with $4 \%$ paraformaldehyde-phosphate-buffered saline overnight, then dehydrated, and embedded in paraffin blocks. After that, a section of $5 \mu \mathrm{m}$ was cut and mounted on slides. The sections were further deparaffinized and hydrated and then stained with hematoxylin-eosin (H\&E) for microscopy. Microphotographs were taken with a DM300 microscope (Leica, Germany). Villus length and crypt depth were performed using Image J software. A minimum of 20 well-orientated villi and associated crypts from at least seven different fields per animal were measured.

\section{RNA Extraction and Quantitative Real-Time Polymerase Chain Reaction Analysis}

Total RNAs from jejunum and ileum samples were isolated using Trizol (Invitrogen, USA) reagent and then treated with DNase I (Invitrogen, USA) according to the instruction of the manufacturer. The concentration of each RNA sample was quantified using NanoDrop 2000 (Nanodrop Technologies, USA). Before reverse transcription, possible contaminations from genomic DNA were eliminated using a PrimeScript RT reagent kit (Takara, Japan). cDNA was synthesized using PrimeScript Enzyme Mix 1, RT Primer Mix, and $5 \times$ PrimerScript Buffer 2 (Takara, Dalian, China). Reverse transcription was conducted at $37^{\circ} \mathrm{C}$ for $15 \mathrm{~min}$ and $85^{\circ} \mathrm{C}$ for $5 \mathrm{~s}$. Genespecific prime sequences (Table 1) were designed using Primer 5.0 software and synthesized by Sangon Biotech Co., Ltd (Shanghai, China). Real-time PCR was performed according to the manufacturer's instructions. Briefly, $1 \mu \mathrm{cDNA}$ template was added to a total volume of $10 \mu \mathrm{l}$ containing $5 \mu \mathrm{l} \mathrm{KAPA}$ SYBR FAST qPCR Master Mix Universal, $0.4 \mu$ l PCR forward primer, $0.4 \mu \mathrm{l}$ PCR reverse primer, $0.2 \mu \mathrm{l}$ ROX low, and $3 \mu \mathrm{l}$ PCR-grade water (Kapa Biosystems, Beijing, China). We used the following protocol: (i) enzyme activation $\left(3 \mathrm{~min}\right.$ at $95^{\circ} \mathrm{C}$ ), (ii) an amplification and quantification program consisting 40 of repeated cycles $\left(3 \mathrm{~s}\right.$ at $95^{\circ} \mathrm{C}$ and $34 \mathrm{~s}$ at $60^{\circ} \mathrm{C}$ ), and (iii) a melting curve program $\left(15 \mathrm{~s}\right.$ at $95^{\circ} \mathrm{C}, 1 \mathrm{~min}$ at $60^{\circ} \mathrm{C}$, and $15 \mathrm{~s}$ at $95^{\circ} \mathrm{C}$ ). Relative expression was calculated between the control group and treatment group by $2^{-\Delta \Delta \mathrm{Ct}}$ method, where $\Delta C_{t}=C_{t}$ (Target) $-C_{t}$ ( $\beta$-actin). $\beta$-actin was chosen as a housekeeping gene to normalize target gene transcript level.
TABLE 1 | Primers used for qPCR assay.

\begin{tabular}{|c|c|c|}
\hline Gene & Accession no. & Sequence $\left(5^{\prime}-3^{\prime}\right)$ \\
\hline \multirow[t]{2}{*}{$\beta$-actin } & NM_007393.5 & F: TGTCCACCTTCCAGCAGATGT \\
\hline & & R: GCTCAGTAACAGTCCGCCTAGAA \\
\hline \multirow[t]{2}{*}{ SOD1 } & NM_011434.2 & F: GTGAACCAGTTGTGTTGTC \\
\hline & & R: ATCACACGATCTTCAATGGA \\
\hline \multirow[t]{2}{*}{ CAT } & NM_009804.2 & F: TCAGGTGCGGACATTCTA \\
\hline & & R: ATTGCGTTCTTAGGCTTCT \\
\hline \multirow[t]{2}{*}{ GPx1 } & NM_001329527.1 & F: ATCAGTTCGGACACCAGA \\
\hline & & R: TTCACTTCGCACTTCTCAA \\
\hline \multirow[t]{2}{*}{ GPx2 } & NM_030677.2 & F: GTGGCGTCACTCTGAGGAACA \\
\hline & & R: CAGTTCTCCTGATGTCCGAACTG \\
\hline \multirow[t]{2}{*}{ TNF- $\alpha$} & NM_013693.3 & F: CATCTTCTCAAAATTCGAGTGACAA \\
\hline & & R: TGGGAGTAGACAAGGTACAACCC \\
\hline \multirow[t]{2}{*}{ IL-1 $1 \beta$} & NM_008361.4 & F: TTCAGGCAGGCAGTATCA \\
\hline & & R: CCAGCAGGTTATCATCATCA \\
\hline
\end{tabular}

$F$, forward; $R$, reverse.

\section{Bacterial 16S rRNA Gene Sequencing and Analysis}

Total genome DNA from colonic digesta was extracted using QIAamp DNA Stool Mini Kit (Qiagen, Germany); then, DNA concentration and purity was monitored on $1 \%$ agarose gels. The V3-V4 region of the bacterial $16 \mathrm{~S}$ ribosomal RNA gene was amplified using a specific primer (338F, 5'-ACTCCTACGGGAGGCAGCAG-3'; 806R, 5' GGACTACHVGGGTWTCTAAT- $3^{\prime}$ ). Amplicons were detected using 2\% agarose gel electrophoresis and purified using the AxyPrep DNA gel extraction kit (Axygen Bioscience, CA, USA) according to the manufacturer's instructions. After having been quantified and purified, the amplicons were sequenced using Illumina MiSeq platform (Illumina, San Diego, CA, USA) at Majorbio Bio-Pharm Technology Co., Ltd. (Shanghai, China) according to standard protocols. The raw reads were deposited into the NCBI Sequence Read Archive database (Accession Number: PRJNA681369). The sequences were analyzed and assigned to operational taxonomic units (OTUs; 97\% identity). Alpha diversity was analyzed using QIIME (Version 1.7.0), which included the calculation of ACE, Chao 1, Shannon, and Simpson indices. Beta diversity was estimated by computing the unweighted Unifrac distance and visualized using principal coordinates analysis (PCoA).

\section{Short-Chain Fatty Acid Analysis}

For short-chain fatty acid (SCFA) analysis, frozen colonic digesta samples $(100 \mathrm{mg})$ were weighed into $1.5-\mathrm{ml}$ centrifuge tubes and

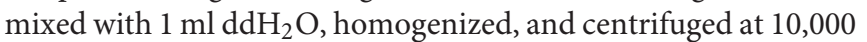
rpm for 10 min under $4^{\circ} \mathrm{C}$. A mixture of the supernatant fluid and $25 \%$ metaphosphoric acid solution ( 0.9 and $0.1 \mathrm{ml}$, respectively) was vortexed for $1 \mathrm{~min}$ and centrifuged at $1,000 \mathrm{rpm}$ for $10 \mathrm{~min}$ under $4^{\circ} \mathrm{C}$ after letting it stand in a 1.5 - $\mathrm{ml}$ centrifuge tube at $4^{\circ} \mathrm{C}$ for over $2 \mathrm{~h}$. The supernatant portion was then filtered through a $0.45-\mu \mathrm{m}$ polysulfone filter and analyzed using Agilent 6890 gas chromatography (Agilent Tecnologies, Inc., Palo Alto, CA, USA). 

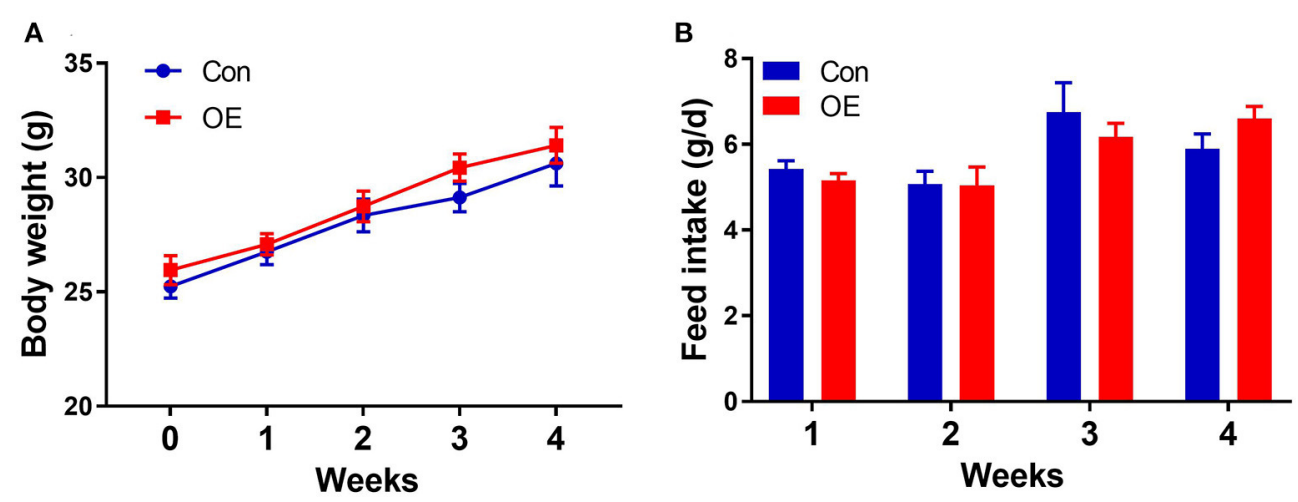

FIGURE 1 | Effects of olive fruit (Canarium album L.) extracts on body weight and feed intake. (A) Body weight. (B) Average daily feed intake. Data were expressed as mean \pm SEM.
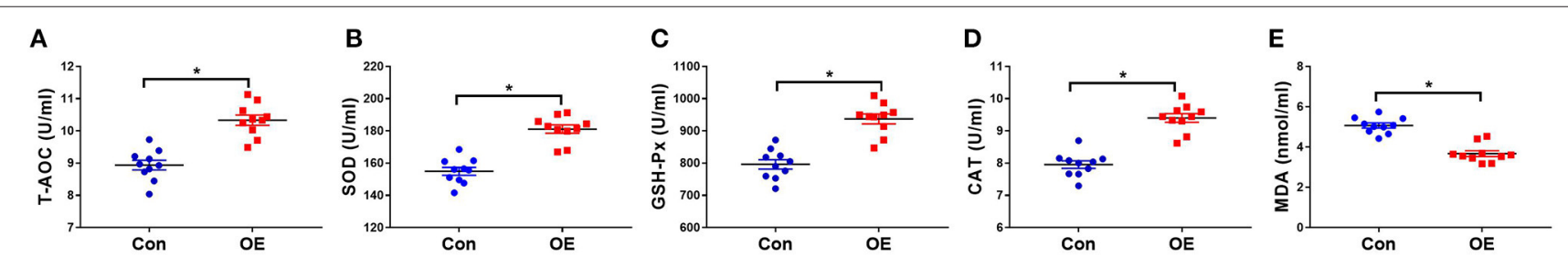

FIGURE 2 | Effects of olive fruit (Canarium album L.) extracts on the serum antioxidant indicators. (A) T-AOC, (B) SOD, (C) GSH-Px, (D) CAT, (E) MDA. Data were expressed as mean \pm SEM. ${ }^{\star} P<0.05$.

\section{Statistical Analysis}

All statistical analyses were performed by using Student's $t$-test (SPSS 21 software). Pearson correlation analysis between the Firmicutes/Bacteroidetes ratio and serum antioxidant indicators (T-AOC, SOD, GSH-Px, CAT, and MDA) was carried out using GraphPad Prism 7.0. Data are expressed as mean \pm SEM. $P$-value $<0.05$ was considered significant.

\section{RESULTS}

\section{Effects of OE Supplement on Body Weight and Feed Intake}

Oral administration with OE for 4 weeks had no significant effects on the body weight $(P>0.05)$ and average daily feed intake in mice compared with the mice in the Con group $(P>$ 0.05; Figure 1).

\section{OE Supplement Enhanced the Serum Antioxidant Capacity in Mice}

The activities of oxidant-antioxidant enzyme and MDA levels are sensitive indicators for oxidative stress. To determine whether $\mathrm{OE}$ affects antioxidant capacity, serum oxidant-antioxidant enzyme activities of T-AOC, SOD, GSH-Px, CAT, and MDA levels were analyzed using test kits. Figure 2 shows that OE treatment significantly increased the T-AOC $(P<0.05)$, increased the activities of SOD $(P<0.05)$, GSH-Px $(P<0.05)$, and CAT $(P<0.05)$, and decreased the level of MDA $(P<0.05)$.

\section{Effects of OE Supplement on Intestinal Morphology}

Intestinal morphology was examined with $\mathrm{H} \& \mathrm{E}$ staining. The villus height, crypt depth, and villus height/crypt depth ratio were measured (Figure 3). In the jejunum, treatment with $\mathrm{OE}$ had no significant effects on the villus height, crypt depth, and villus height/crypt depth ratio in mice $(P>0.05$; Figure 3B). In the ileum, the villus height and crypt depth had no significant difference between the two groups $(P>0.05$; Figure $3 \mathrm{C})$. However, the villus height/crypt depth ratio was significantly higher in the $\mathrm{OE}$ group than in the Con group $(P<0.05$; Figure 3C).

\section{OE Altered Small Intestinal Antioxidant Capacity}

Next, we analyzed the mRNA expression of genes associated with antioxidant capacity in the ileum and jejunum, including $S O D 1$, Gpx1, Gpx2, and CAT to examine the molecular mechanism of $\mathrm{OE}$ administration in enhancing antioxidant capacity. In the jejunum, compared with the Con group, OE treatment significantly down-regulated the mRNA expression of SOD1, $C A T$, Gpx1, and Gpx2 $(P<0.05$; Figure 4A). Similarly, in the ileum, the mRNA expression of SOD1, CAT, and Gpx2 was markedly lower in the OE group than in the Con group $(P<0.05$; Figure 4B). Besides that, the results showed that, in the ileum and jejunum, the MDA level was significantly higher $(P<0.05)$, while the T-AOC was significantly lower in the OE group compared with that in the Con group $(P<0.05$; Figures $4 \mathrm{C}-\mathbf{F})$. 


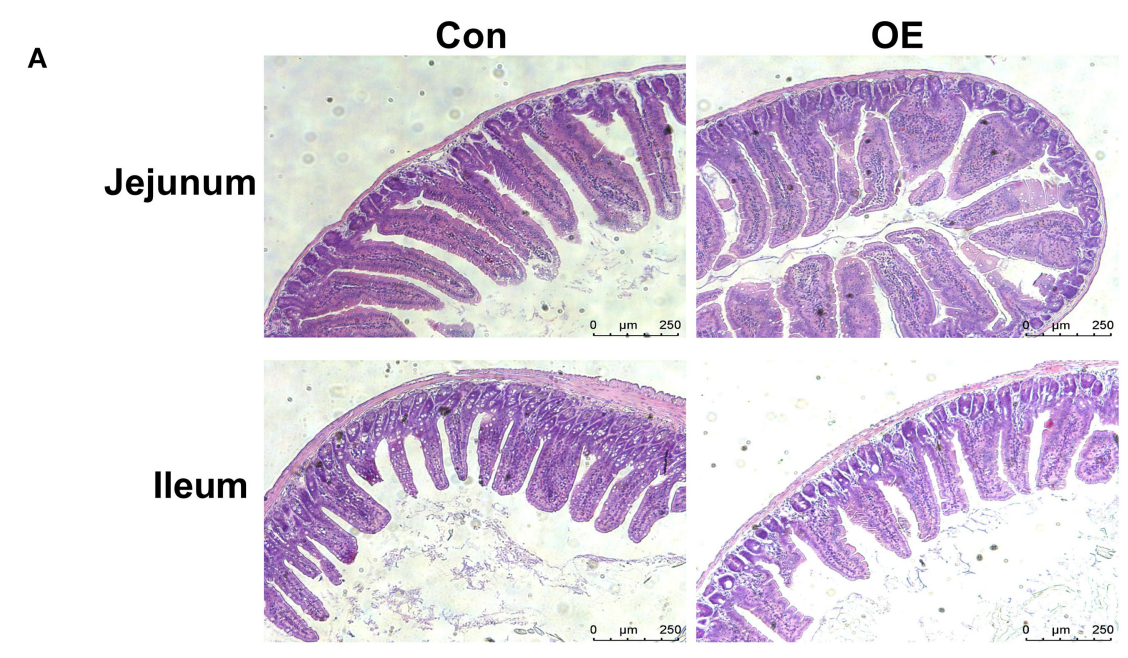

\section{B Jejunum}
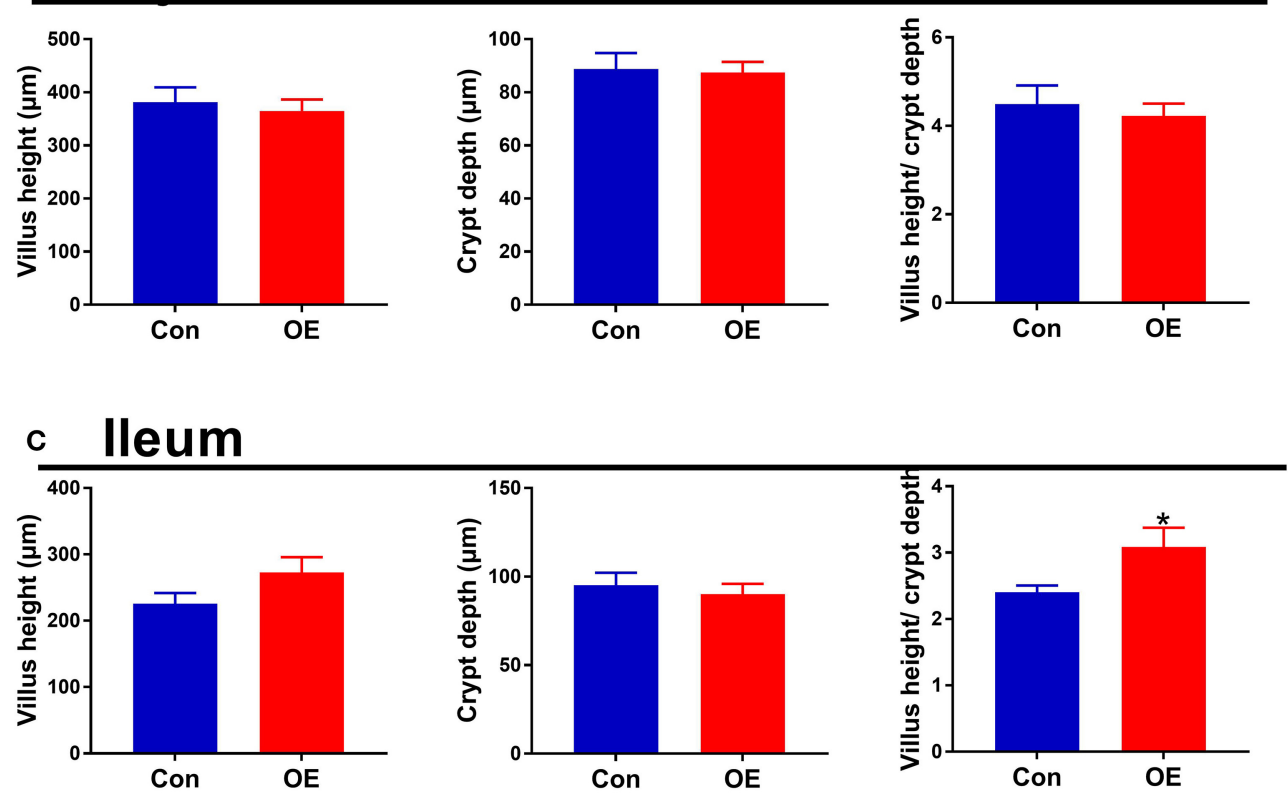

FIGURE 3 | Effects of olive fruit (Canarium album L.) extracts on jejunal and ileal morphology. (A) Representative images of H\&E staining in the jejunum and ileum. (B) Jejunal villus height, crypt depth, villus height/crypt depth. (C) lleal villus height, crypt depth, villus height/crypt depth. Data were expressed as mean \pm SEM. ${ }^{\star} P<0.05$.

\section{OE Supplement Altered the Expression and Levels of Pro-inflammatory Cytokines in the Small Intestine}

Oxidative stress is often involved in inducing inflammatory responses. Thus, the anti-inflammatory capacity of $\mathrm{OE}$ was also tested by analyzing the mRNA expression of pro-inflammatory cytokines (TNF- $\alpha$ and IL-1 $\beta$ ) in the intestine. The results showed that, in the jejunum, oral administration of OE markedly down-regulated the mRNA levels of TNF- $\alpha$ and IL-1 $\beta(P<$ 0.05 ; Figure $5 \mathrm{~A})$. OE administration showed a likely significant decrease in IL-1 $\beta$ mRNA expression in the ileum compared with the Con group $(P<0.05$; Figure 5B). However, the ELISA results showed that, in the jejunum and ileum, the pro-inflammatory cytokine (IL-6, IL-1 $\beta$, TNF- $\alpha$, and IFN- $\gamma$ ) levels were significantly higher in the OE group than in the Con group $(P<0.05$; Figures 5C,D).

\section{OE Supplement Altered the Composition of Colonic Microbiota Community}

To study the effect of $\mathrm{OE}$ supplementation on large intestinal microbiota composition, the colonic chyme microflora was analyzed by sequencing V3 + V4 regions of 16S rRNA genes. 


\section{A}

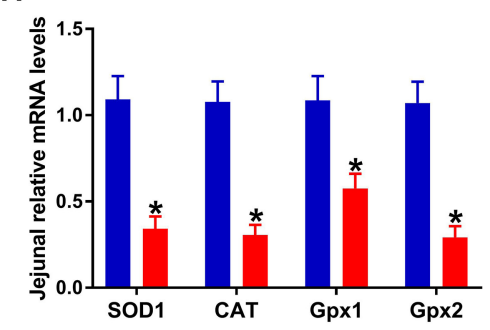

C

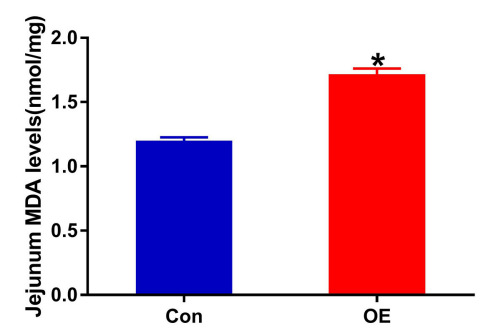

E

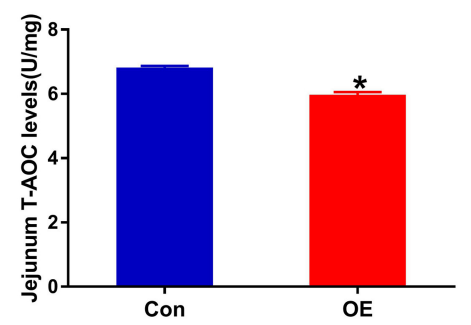

B

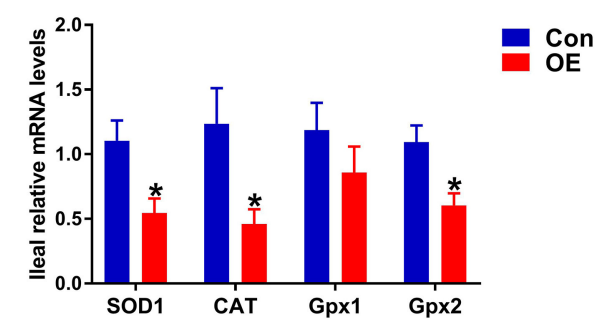

D

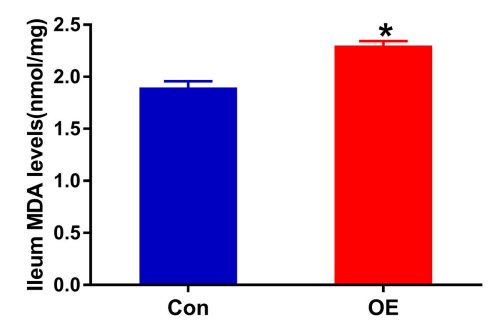

$\mathbf{F}$

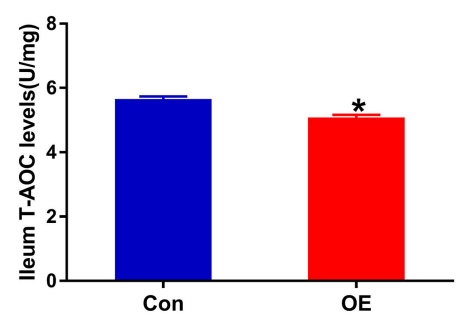

FIGURE 4 | Effects of olive fruit (Canarium album L.) extracts on the jejunal and ileal antioxidant capacities. (A) Jejunum mRNA expression levels of antioxidant enzymes. (B) lleum mRNA expression levels of antioxidant enzymes. (C) Jejunum MDA levels. (D) lleum MDA levels. (E) Jejunum T-AOC levels. (F) Ileum T-AOC levels. Data were expressed as mean \pm SEM. ${ }^{*} P<0.05$.

After removing the low-quality sequences, a total of 1,184,959 clean tags were clustered into OTUs based on $97 \%$ identity. The dilution curves showed that the end of the curve tends to be flat, indicating that the amount and depth of high-throughput sequencing data is reliable (Figure 6A). To identify the microbial $\alpha$-diversity, ACE and Chao 1 indexes were examined for the community richness, and Shannon and Simpson were examined for the community diversity. As shown in Figure 6B, OE treatment significantly decreased the Shannon index $(P<$ 0.05), while it had a little effect on the ACE, Chao 1, and Simpson indexes compared to the Con group. The Venn diagram shows that there are 464 common OTUs between the Con and OE groups. Meantime, the Con and OE groups contained individual 50 and 101 OTUs, respectively (Figure 6C). To further understand the microbial composition between the Con and OE groups, we evaluated $\beta$-diversity using PCoA based on unweighted Unifrac. The results showed that the microbial community structure in the OE group significantly differed from that in the control group (Figure 6C).

The overall microbial composition in the Con and OE groups differed at the phylum and genus levels. Linear discriminant analysis effect size (LEfSe) analysis was performed to evaluate the differentially expressed bacteria. Of note is the fact that Staphylococcales and Bacillaceae were shown to be enriched in the OE treatment group (Figures 7A,B). The relative abundance results showed that, at the phylum level, OE supplement notably enhanced the Firmicutes/Bacteroidetes ratio $(P<0.05)$, while it did not affect the relative abundance of Firmicutes and Bacteroidetes, respectively $(P>0.05$; Figure 7C). At the genus level, $\mathrm{OE}$ tended to decrease the relative abundance of Candidatus_Arthromitus, but the difference was not significant $(P>0.05)$. However, the relative abundance of unclassified_f_Lachnospiraceae was significantly lower in the $\mathrm{OE}$ group than that in the control group $(P<0.05$; Figure 7D).

\section{The Association Analysis Between OE Supplement-Induced Alterations in Colonic Microbiota and Serum Antioxidant Capacity}

To investigate whether the alteration in gut microbiota is associated with the antioxidant effects of OE, we performed a correlation analysis using the Firmicutes/Bacteroidetes ratio and serum antioxidant indicators (T-AOC, SOD, GSH-Px, CAT, and MDA). As shown in Figure 8, there was a negative correlation between the Firmicutes/Bacteroidetes ratio and the 
A

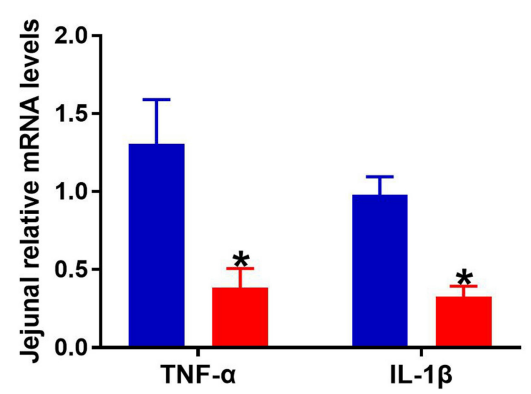

C

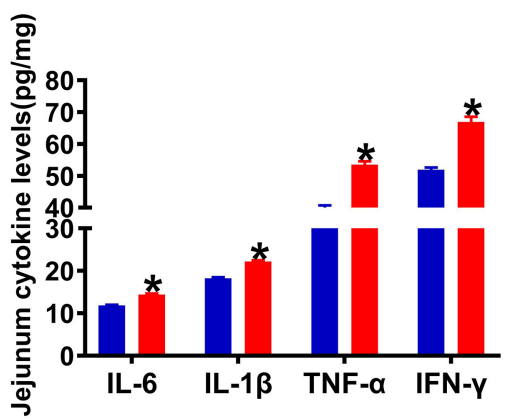

B

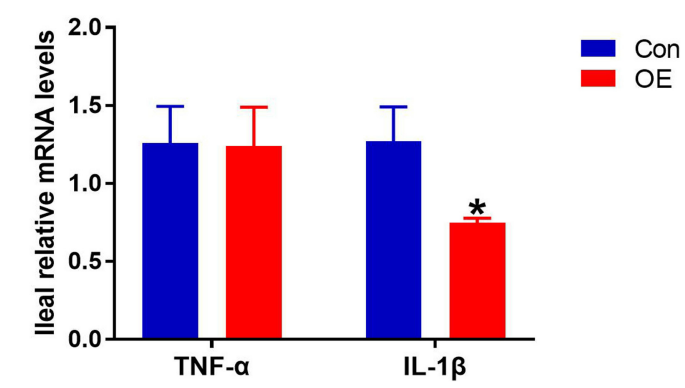

D

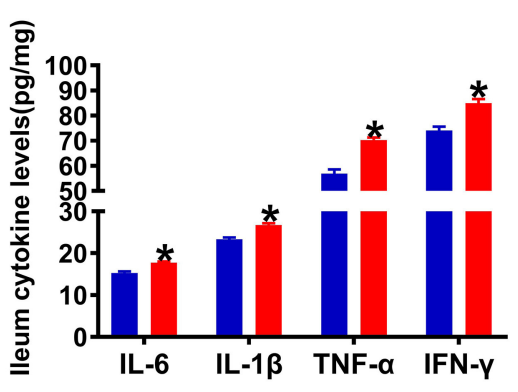

FIGURE 5 | Effects of olive fruit (Canarium album L.) extracts on the jejunal and ileal pro-inflammatory cytokines. (A) Jejunal relative mRNA levels of cytokines. (B) lleal relative mRNA levels of cytokines. (C) Jejunal cytokine levels. (D) lleal cytokine levels. Data were expressed as mean \pm SEM. ${ }^{*} P<0.05$.

level of serum MDA $(P<0.05$; Figure $8 \mathrm{E})$ as well as a positive correlation between the Firmicutes/Bacteroidetes ratio and the activity of serum GSH-Px $(P<0.05$; Figure $8 \mathrm{C})$. Moreover, there was a positive correlation trend between the Firmicutes/Bacteroidetes ratio and the activities of serum T-AOC $(0.05<P<0.1)$, SOD $(0.05<P<0.1)$, and CAT $(0.05<$ $P<0.1$; Figures 8A,B,D). In addition, heat map revealed the correlation between the gut microbial population at the genus level and the serum antioxidant indicators (T-AOC, SOD, GSH$\mathrm{Px}, \mathrm{CAT}$, and MDA). The data showed that the relative abundance of Colidextribacter was positively correlated with serum MDA level and negatively correlated with serum T-AOC, SOD, and GSH-Px levels $(P<0.05$; Figure 9). The relative abundance of Alloprevotella was found to be likely markedly negatively correlated with the serum T-AOC $(P<0.05$; Figure 9).

\section{OE Supplementation Had No Effects on Colonic SCFAs}

Since the supplement of OE altered colonic microbiota composition and structure and SCFAs as the metabolites of microbiota, we investigated the SCFA content in the colon. The results showed that oral administration of OE had no significant effect on the levels of SCFAs, including acetic acid, propionic acid, and butyrate, in the colon $(P>0.05$; Figure 10).

\section{DISCUSSION}

Studies being conducted both in animal models and humans have revealed the significant role of oxidative stress in the pathogenesis of various diseases, including neurodegenerative and metabolic diseases and cancer (3,33-35). Emerging evidence has highlighted the beneficial bioactivity of olive fruit, pomace, leaf extracts, and olive oils because of the many bioactive polyphenolic compounds, which have various health-promoting potentials including antioxidant, anti-inflammation, and antibacteria (36-38). In this study, we have demonstrated that oral administration of $\mathrm{OE}$ was able to enhance the antioxidative capacity as well as the anti-inflammatory activity in mice, which may be associated with the changes of gut microbiota induced by OE treatment.

Investigations have shown that olive extracts contain polyphenols which exhibit powerful antioxidant and antiinflammatory effects on humans and animals $(39,40)$. For instance, numerous evidence has demonstrated that olive extracts can ameliorate oxidative stress and inflammation in different cells, such as colon cancer cells (41), kidney cells (42, 43), and renal cells (44). In addition, oral administration of olive extracts could alleviate the lipopolysaccharide (LPS)induced oxidative stress and inflammatory responses as shown by attenuating the decreased levels of brain GSH and increased levels of brain MDA and serum TNF- $\alpha$ in mice (12). Similarly, in this study, we found that oral administration of OE increased the serum T-AOC and the activities of antioxidant enzymes, including SOD, GSH-Px, and CAT, and decreased the MDA levels in mice. SOD, GSH-Px, and CAT are generally regarded as the primary antioxidant enzyme defense system in animals and humans (45). SOD can catalyze superoxide into oxygen and hydrogen peroxide (45). CAT 


\section{A dilution curve}
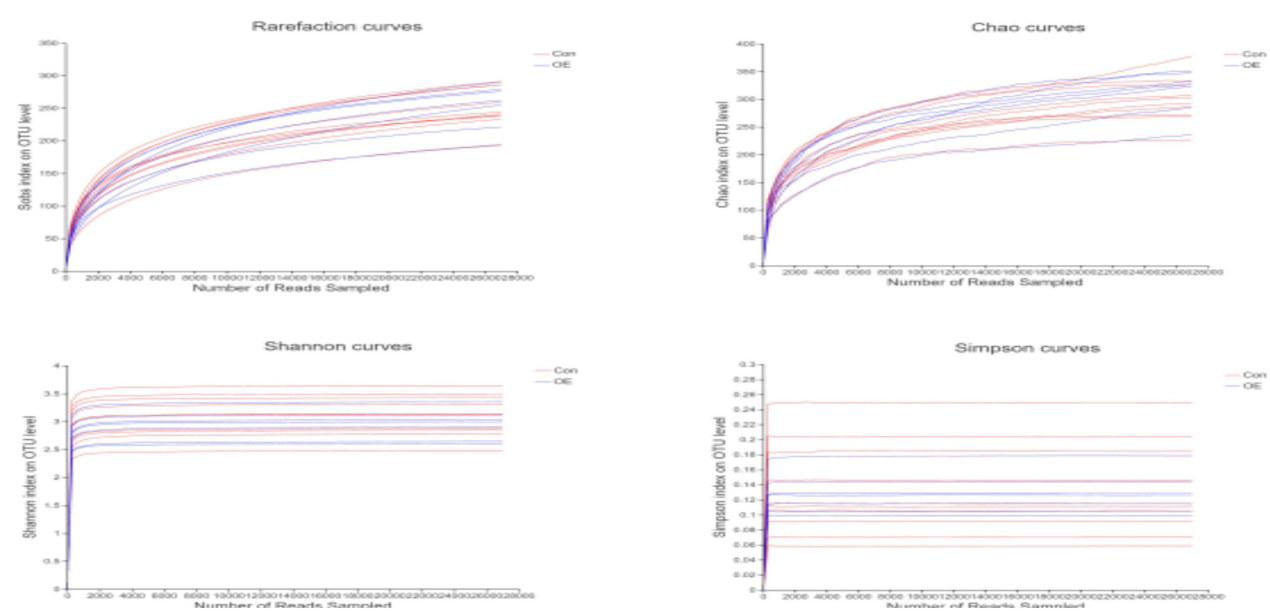

B

a-diversity
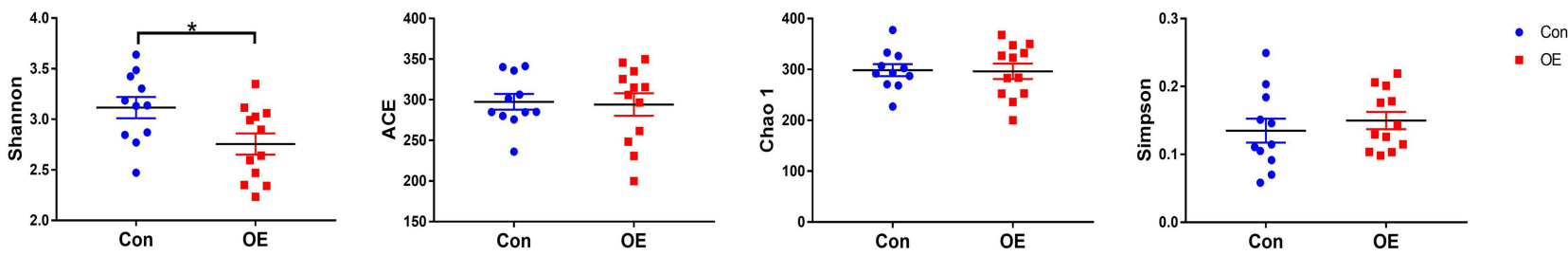

C
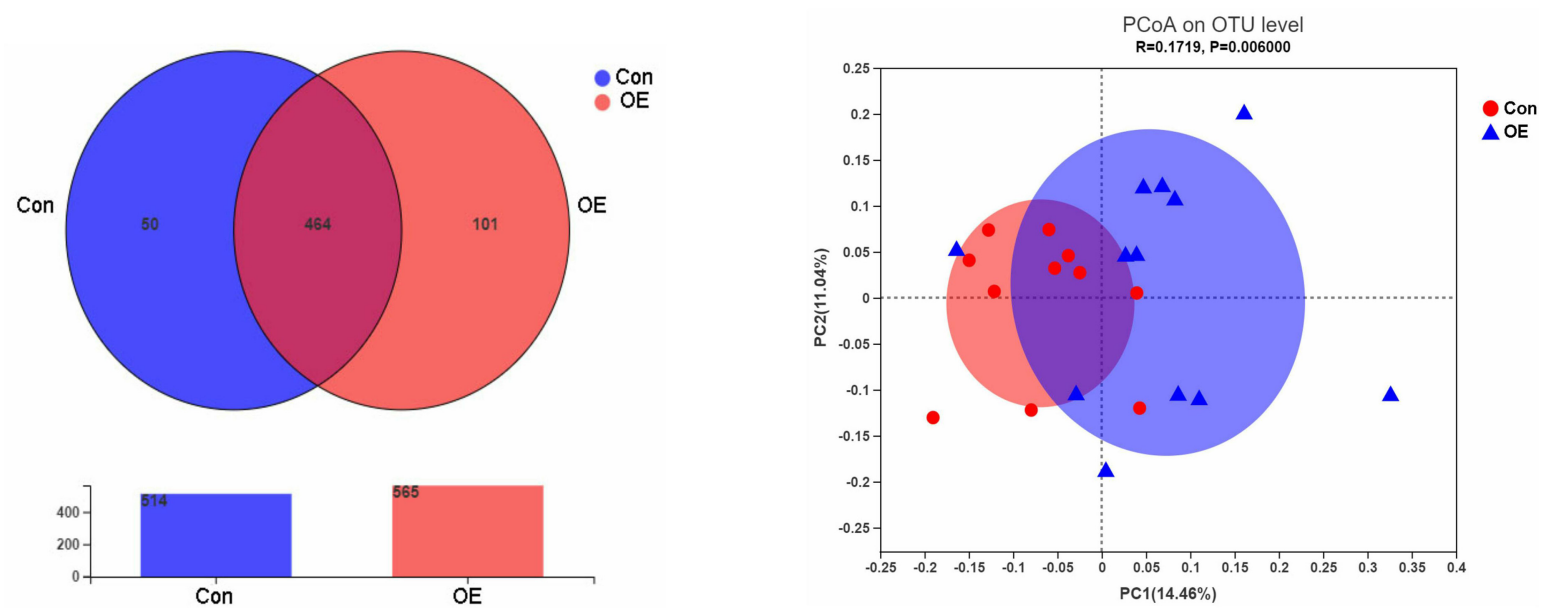

FIGURE 6 | Effects of olive fruit (Canarium album L.) extracts on the colonic microbial diversity. (A) Dilution curve. (B) $\alpha$-diversity. (C) Venn diagram. Data were expressed as mean \pm SEM.

has the ability to scavenge hydrogen peroxide into oxygen and water (45). GSH-Px can catalyze hydrogen peroxide into water (46). MDA is one of the products of lipid peroxidation, and it is an important indicator of oxidative stress status in the body (47). These results of serum indicators suggested that $\mathrm{OE}$ supplement could enhance the antioxidant ability in mice.
The intestine, a vital organ responsible for nutrient digestion and absorption and a major site of host immunity, is highly susceptible to oxidative stress, which leads to gut dysfunction and body disorders $(2,11,48,49)$. It is well-documented that dietary polyphenols can be absorbed in the small intestine (50) and exhibit antioxidant effects by scavenging oxidant chemical species as well as altering the levels and activities of antioxidant 


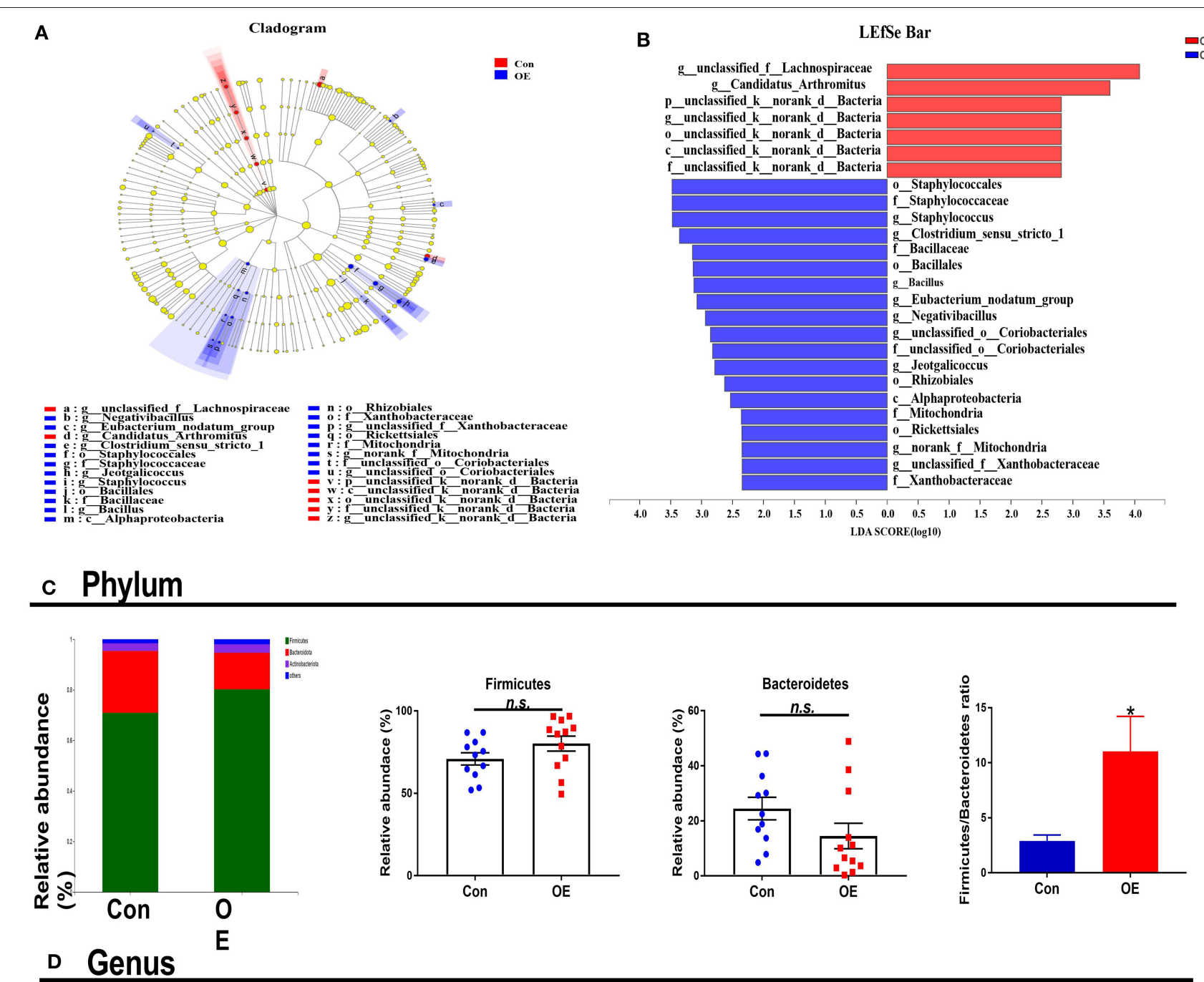

\section{Genus}
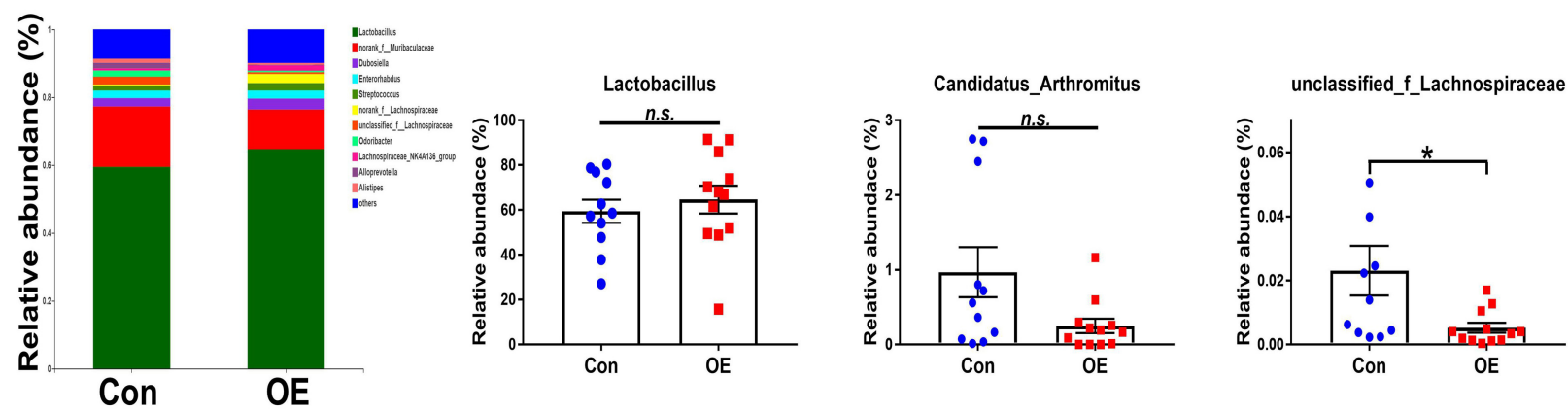

FIGURE 7 | Effects of olive fruit (Canarium album L.) extracts on the colonic microbial composition. (A,B) LEfSe analysis. (C) Phylum level. (D) Genus level. Data were expressed as mean \pm SEM. ${ }^{*} P<0.05$; n.s., not statistically significant.

enzymes (11). On the other hand, polyphenolic compounds are commonly recognized as xenobiotics by the enterocytes, which will induce stress (51). Thus, we investigated the effects of $\mathrm{OE}$ on oxidative stress in the small intestine of mice. Based on the activities of serum antioxidation enzymes, we then detected the transcript levels of Nrf2-associated antioxidant enzymes in the intestine, including Sod, Cat, and Gpx (52). In mice with severe oxidative stress status, previous studies have shown that extracts from olive oil and olive leaf ameliorated oxidative stress by up-regulating the Nrf2/ARE antioxidant signaling pathways $(53,54)$. Nrf2, a transcription factor, is activated and translocated to the nucleus during oxidative stress and enhances the expressions of Nrf2-related antioxidant enzymes (55). Interestingly, in this study, the qPCR results showed that OE treatment decreased jejunal and ileal Sod, Cat, Gpxl, and Gpx2 expressions. Consistently, our study also indicated that $\mathrm{OE}$ 

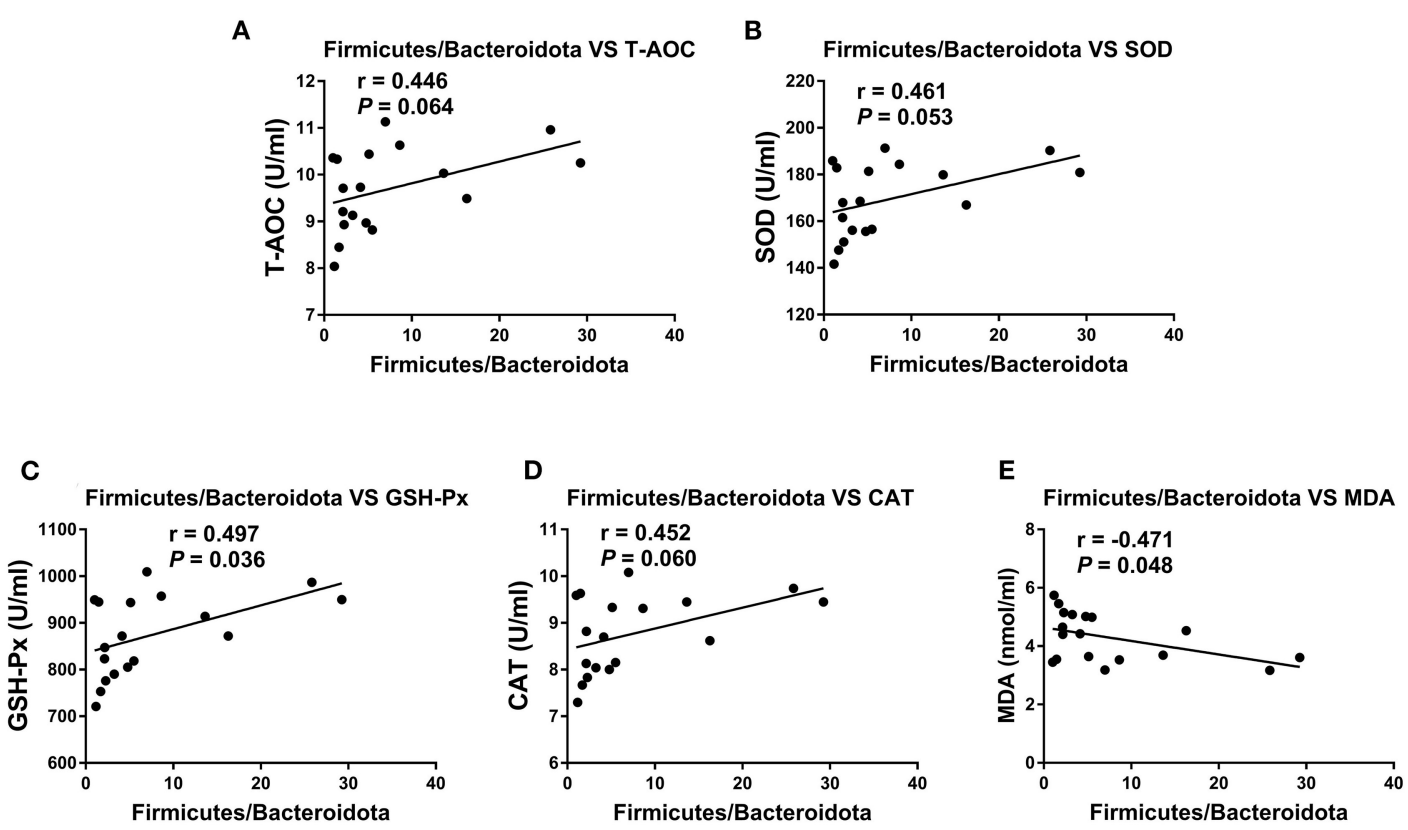

FIGURE 8 | Pearson correlation analyses between the colonic Firmicutes/Bacteroidetes ratio and serum antioxidant indicators and liver and kidney mRNA expression levels of TNF- $\alpha$ and IL-1 $\beta$. (A) Correlation analyses between the Firmicutes/Bacteroidetes ratio and serum T-AOC. (B) Correlation analyses between the Firmicutes/Bacteroidetes ratio and serum SOD. (C) Correlation analyses between the Firmicutes/Bacteroidetes ratio and serum GSH-Px. (D) Correlation analyses between the Firmicutes/Bacteroidetes ratio and serum CAT. (E) Correlation analyses between the Firmicutes/Bacteroidetes ratio and serum MDA.

treatment increased the MDA concentration and decreased TAOC in the jejunum and ileum. We speculated that this may be because $\mathrm{OE}$ acted as a xenobiotic, which can induce mild stress in the small intestine of mice (51). However, this slight oxidative stress had no negative effect on the small intestine, which was confirmed by the intestinal morphology without change after OE treatment.

In the meantime, amounts of inflammation-related transcription factors were activated under oxidative stress state, which will initiate the inflammatory process and lead to the increased production of pro-inflammatory cytokines (11). TNF- $\alpha$ and IL- $1 \beta$ are usually considered to be the two key regulators of pro-inflammatory response, which are involved in promoting inflammation and causing tissue damage (56). A previous study has demonstrated that olive extracts had anti-IL-1 $\beta$ activity in humans (57). An in vitro study also found that polyphenol-rich olive extracts decreased the mRNA expression of pro-inflammatory cytokines (58). Consistently, in this study, we also found that $\mathrm{OE}$ treatment decreased the mRNA expressions of TNF- $\alpha$ and IL- $1 \beta$ in the jejunum and ileum. However, interestingly, we found that the levels of inflammatory cytokines in the small intestine (jejunum and ileum) were all significantly increased, which may be caused by the OE-induced mild oxidative stress. Furthermore, we suggested that the absorption of $\mathrm{OE}$ in the small intestine caused down-regulation in mRNA expression of pro-inflammatory cytokines (TNF- $\alpha$ and IL-1 $\beta$ ) to play anti-inflammatory activity. Based on the above-mentioned data, we speculated that $\mathrm{OE}$ tends to enhance the anti-inflammatory capacity by down-regulating the expressions of pro-inflammatory cytokines.
According to emerging evidence, the majority of dietary polyphenols (including HT) are metabolized by the colonic microbiota (59-61). Meanwhile, in vivo studies showed that both olive extracts with complex composition and individual phenolic compounds purified from olive extracts had the ability to modulate bacterial growth and reproduction in the intestine $(21,22)$. However, little is known about the effects of $\mathrm{OE}$ administration on the gut microbiota and whether the gut microbiota associates with the antioxidant and antiinflammatory effects of OE in mice. Therefore, we analyzed the diversity of the colonic microbial community in OE-treated mice. The results showed that oral administration of OE supplement decreased the Shannon index, suggesting that OE lowered the $\alpha$-diversity of colonic microbiota, which might be due to the anti-bacterial effects of OE. Additionally, LEfSe results showed that Staphylococcales are enriched in the OE group, suggesting that the increased Staphylococcales after OE treatment may be one of the reasons for the enhanced antioxidant capacity. Consistently, another study reported that Staphylococcales can reduce endogenous and exogenous oxidative stress (62). Besides that, at the phylum level, OE treatment increased the Firmicutes/Bacteroidetes ratio. As the main metabolites of colonic microbiota, the increased Firmicutes/Bacteroidetes ratio did not change the composition of SCFAs significantly in this study, which is in agreement with the previous study (63). To further examine whether OE-induced microbial alteration associates with its antioxidant effects, we conduct correlation analyses between the Firmicutes/Bacteroidetes ratio and serum antioxidant enzyme activities and MDA level by Pearson correlation analysis. Surprisingly, the results showed that the 

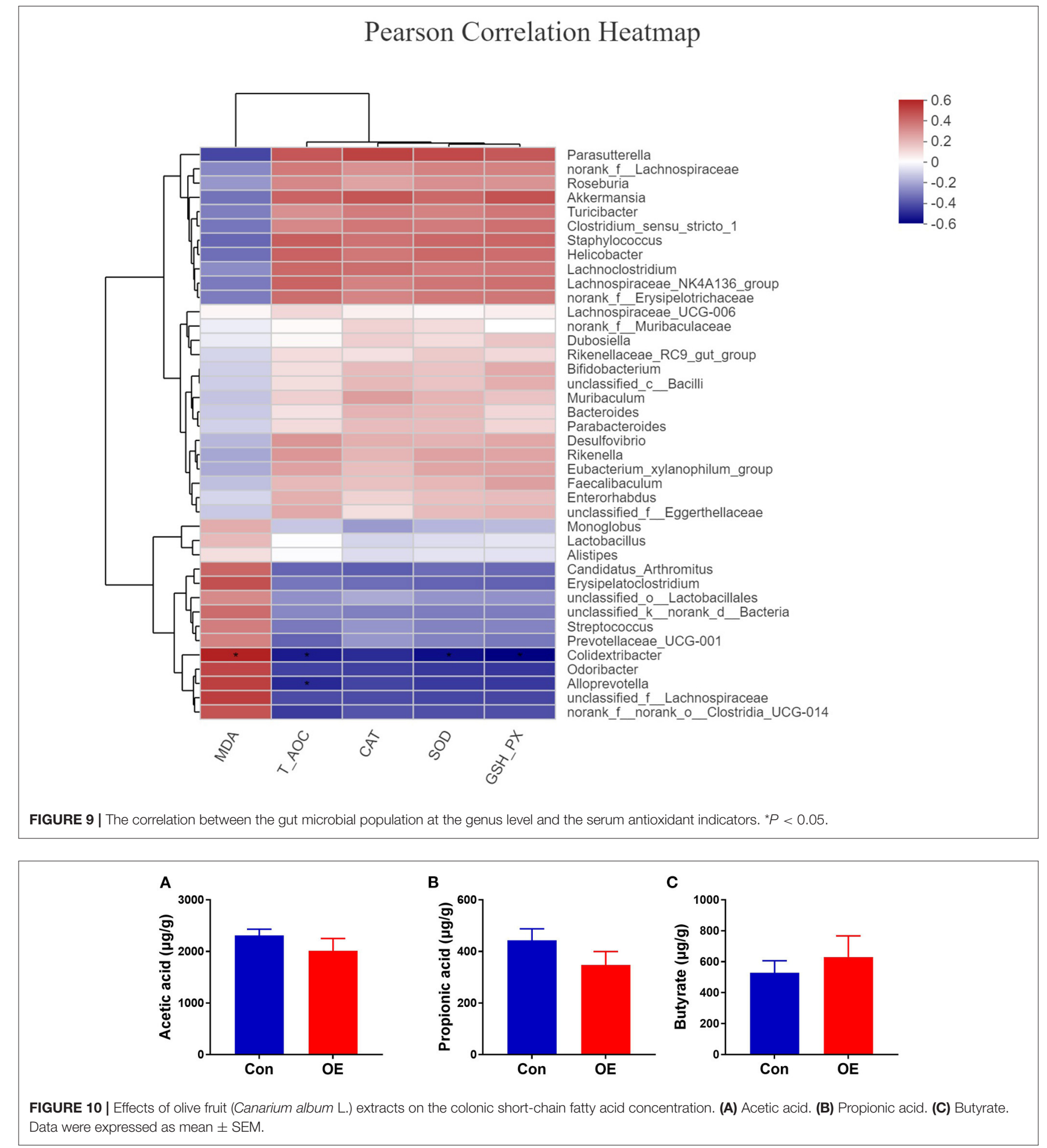

Firmicutes/Bacteroidetes ratio was negatively correlated with the serum MDA content and positively correlated with the serum GSH-Px activity. In piglets and sows, studies have indicated that oxidative stress has a direct correlation with gut microbiota (64-67). This information in our study contributed to the new understanding of the OE-enhanced antioxidant capacity, at least in part, due to alterations in the gut microbiota in mice. In addition, Firmicutes are considered to be involved in maintaining intestinal barrier integrity, which plays a key role in modulating host inflammation (68). Meanwhile, bacteria in phylum Bacteroidetes have the ability to release LPS, which then leads to higher inflammatory responses (69). So, a decreased 


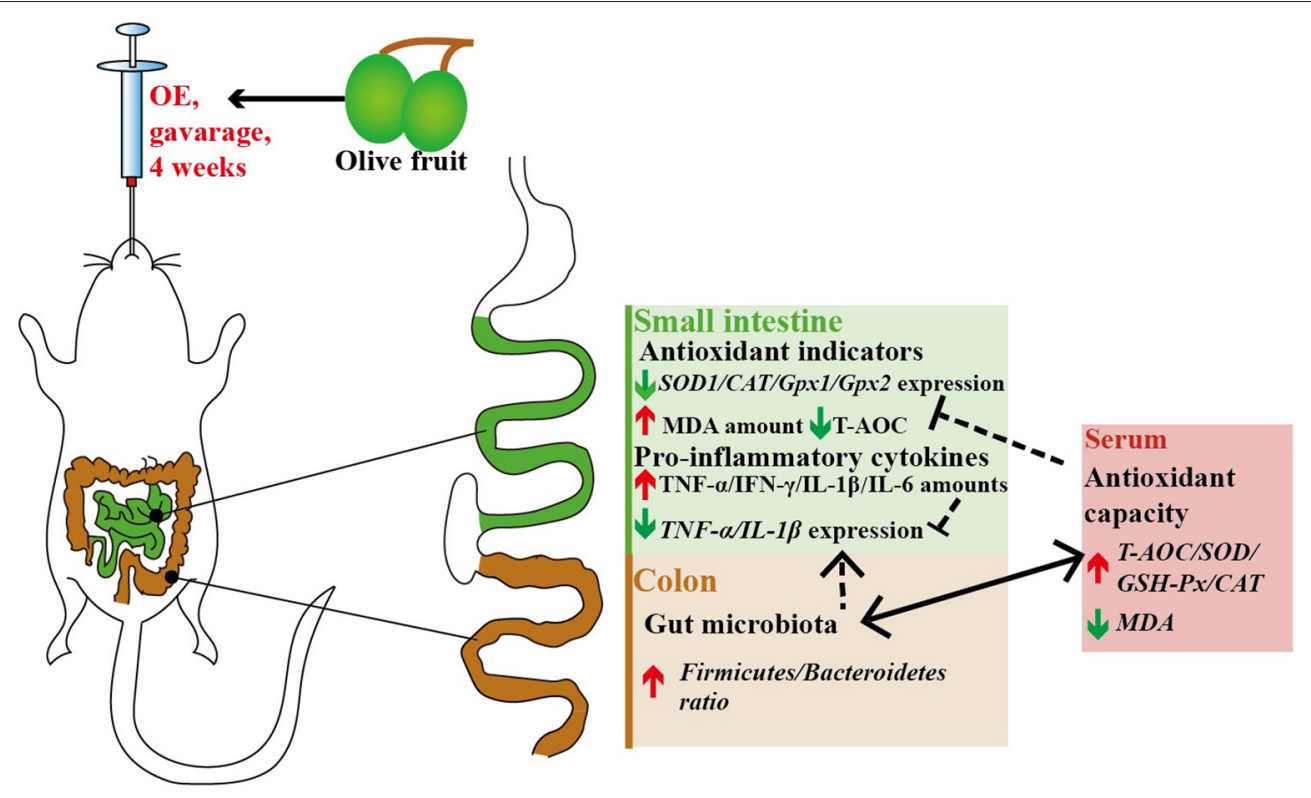

FIGURE 11 | Antioxidant and anti-inflammatory effects of olive fruit (Canarium album L.) extracts administration in mice: a possible mechanism.

proportion of Bacteroidetes may be related to lower inflammatory factors, which is consistent with our results. In humans, healthy adults who are more resistant to pathogens have a higher Firmicutes/Bacteroidetes ratio than infants and the elderly (70). Similarly, in piglets, a higher Firmicutes/Bacteroidetes ratio is associated with enhanced oxidative response and lower inflammation and infection risk (71-73). Therefore, it appears reasonable to speculate that $\mathrm{OE}$ administration exhibits antioxidant and anti-inflammatory effects closely associated with the increased Firmicutes/Bacteroidetes ratio in the colon of mice. At the genus level, Alloprevotella was highly negatively correlated with serum T-AOC. Similarly, Zhang et al. showed that OE can ameliorate oxidative stress and reduce the relative abundance of fecal Alloprevotella in LPS-challenged piglets (74), suggesting that Alloprevotella may play a role in enhancing antioxidant capacity. In addition, we also found that Colidextribacter is highly correlated with the levels of MDA, T-AOC, SOD, and GSH-Px in serum, which however needs further investigation.

Although OE contains various polyphenols, current evidence indicates that the beneficial health properties of OE are mainly related to HT, which needs more and further investigation (24-26). As summarized in Figure 11, in conclusion, our data provide a new insight that oral treatment with $\mathrm{OE}$ can improve the antioxidant capacity by enhancing the circulating activities of antioxidant enzymes, and the most important is that the improved antioxidant capacity is connected to the increased colonic Firmicutes/Bacteroidetes ratio as well as the change of Alloprevotella and Colidextribacter in mice.

\section{DATA AVAILABILITY STATEMENT}

The data used to support the findings of this study are available from the corresponding author upon request. Sequencing raw data on colonic microbiota of mice were deposited into the NCBI Sequence Read Archive (SRA) database (PRJNA681369).

\section{ETHICS STATEMENT}

The studies involving human participants were reviewed and approved by Experimental Animal Welfare and Ethical Committee of the Institute of Animal Science, Chinese Academy of Agricultural Sciences. The patients/participants provided their written informed consent to participate in this study. The animal study was reviewed and approved by Experimental Animal Welfare and Ethical Committee of the Institute of Animal Science, Chinese Academy of Agricultural Sciences. Written informed consent was obtained from the owners for the participation of their animals in this study.

\section{AUTHOR CONTRIBUTIONS}

MW and SZ conducted the study. MW, RZ, SZ, and FW helped to perform the experiment. MW, LC, and RZ helped to write the paper. BY and $\mathrm{HZ}$ designed the experiment and revised the manuscript. All authors contributed to the article and approved the submitted version.

\section{FUNDING}

This study was supported by the Major Scientific Research Tasks for Scientific and Technological Innovation Projects of the Chinese Academy of Agricultural Sciences (CAAS-ZDRW202006-02), National Natural Science Foundation of China (31702119), National Key Research and Development Program of China (2016YFD0500501), and China Agriculture Research System (CARS-41). 


\section{REFERENCES}

1. Fuccelli R, Fabiani R, Rosignoli P. Hydroxytyrosol exerts anti-inflammatory and anti-oxidant activities in a mouse model of systemic inflammation. Molecules. (2018) 23:3212. doi: 10.3390/molecules23123212

2. Yin J, Liu M, Ren W, Duan J, Yang G, Zhao Y, et al. Effects of dietary supplementation with glutamate and aspartate on diquat-induced oxidative stress in piglets. PLOS ONE. (2015) 10:e0122893. doi: 10.1371/journal.pone.0122893

3. Matyas C, Haskó G, Liaudet L, Trojnar E, Pacher P. Interplay of cardiovascular mediators, oxidative stress and inflammation in liver disease and its complications. Nat Rev Cardiol. (2021) 18:117-35. doi: 10.1038/s41569-020-0433-5

4. He Y, Sang Z, Zhuo Y, Wang X, Guo Z, He L, et al. Transport stress induces pig jejunum tissue oxidative damage and results in autophagy/mitophagy activation. J Anim Physiol Anim Nutr (Berl). (2019) 103:1521-9. doi: 10.1111/jpn.13161

5. Lykkesfeldt J, Svendsen O. Oxidants and antioxidants in disease: oxidative stress in farm animals. Vet J. (2007) 173:502-11. doi: 10.1016/j.tvjl.2006.06.005

6. Forrester SJ, Kikuchi DS, Hernandes MS, Xu Q, Griendling KK. Reactive oxygen species in metabolic and inflammatory signaling. Circ Res. (2018) 122:877-902. doi: 10.1161/circresaha.117.311401

7. Rojo de la Vega M, Chapman E, Zhang DD. NRF2 and the hallmarks of cancer. Cancer Cell. (2018) 34:21-43. doi: 10.1016/j.ccell.2018.03.022

8. Nguyen M, Wong YC, Ysselstein D, Severino A, Krainc D. Synaptic, mitochondrial, and lysosomal dysfunction in Parkinson's disease. Trends Neurosci. (2019) 42:140-9. doi: 10.1016/j.tins.2018.11.001

9. Milic M, Frustaci A, Del Bufalo A, Sánchez-Alarcón J, Valencia-Quintana $\mathrm{R}$, Russo $\mathrm{P}$, et al. DNA damage in non-communicable diseases: a clinical and epidemiological perspective. Mutat Res. (2015) 776:11827. doi: 10.1016/j.mrfmmm.2014.11.009

10. Mishra V, Banga J, Silveyra P. Oxidative stress and cellular pathways of asthma and inflammation: therapeutic strategies and pharmacological targets. Pharmacol Ther. (2018) 181:169-82. doi: 10.1016/j.pharmthera.2017.08.011

11. Hussain T, Tan B, Yin Y, Blachier F, Tossou MC, Rahu N. Oxidative stress and inflammation: what polyphenols can do for us? Oxid Med Cell Longevity. (2016) 2016:7432797. doi: 10.1155/2016/7432797

12. Gupta A, Vij G, Chopra K. Possible role of oxidative stress and immunological activation in mouse model of chronic fatigue syndrome and its attenuation by olive extract. J Neuroimmunol. (2010) 226:37. doi: 10.1016/j.jneuroim.2010.05.021

13. Yeh YT, Cho YY, Hsieh SC, Chiang AN. Chinese olive extract ameliorates hepatic lipid accumulation in vitro and in vivo by regulating lipid metabolism. Sci Rep. (2018) 8:1057. doi: 10.1038/s41598-018-19553-1

14. Erol Ö, Arda N, Erdem G. Phenols of virgin olive oil protects nuclear DNA against oxidative damage in HeLa cells. Food Chem Toxicol. (2012) 50:3475-9. doi: 10.1016/j.fct.2012.07.048

15. Maalej A, Mahmoudi A, Bouallagui Z, Fki I, Marrekchi R, Sayadi S. Olive phenolic compounds attenuate deltamethrin-induced liver and kidney toxicity through regulating oxidative stress, inflammation and apoptosis. Food Chem Toxicol. (2017) 106:455-65. doi: 10.1016/j.fct.2017.06.010

16. Hermans N, Van der Auwera A, Breynaert A, Verlaet A, De Bruyne T, Van Gaal L, et al. A red yeast rice-olive extract supplement reduces biomarkers of oxidative stress, OxLDL and Lp-PLA(2), in subjects with metabolic syndrome: a randomised, double-blind, placebo-controlled trial. Trials. (2017) 18:302. doi: 10.1186/s13063-017-2058-5

17. Flamminii F, Di Mattia CD, Difonzo G, Neri L, Faieta M, Caponio F, et al. From by-product to food ingredient: evaluation of compositional and technological properties of olive-leaf phenolic extracts. J Sci Food Agric. (2019) 99:6620-7. doi: 10.1002/jsfa.9949

18. Weinbrenner T, Fitó M, de la Torre R, Saez GT, Rijken P, Tormos C, et al. Olive oils high in phenolic compounds modulate oxidative/antioxidative status in men. J Nutr. (2004) 134:2314-21. doi: 10.1093/jn/134.9.2314

19. Çoban J, Öztezcan S, Dogru-Abbasoglu S, Bingül I, Yeşil-Mizrak K, Uysal M. Olive leaf extract decreases age-induced oxidative stress in major organs of aged rats. Geriatr Gerontol Int. (2014) 14:996-1002. doi: 10.1111/ggi.12192

20. De Bruno A, Romeo R, Fedele FL, Sicari A, Piscopo A, Poiana M. Antioxidant activity shown by olive pomace extracts. J Environ Sci Health B. (2018) 53:526-33. doi: 10.1080/03601234.2018.1462928
21. Kishikawa A, Ashour A, Zhu Q, Yasuda M, Ishikawa H, Shimizu K. Multiple biological effects of olive oil by-products such as leaves, stems, flowers, olive milled waste, fruit pulp, and seeds of the olive plant on skin. Phytother Res. (2015) 29:877-86. doi: 10.1002/ptr.5326

22. Gilling DH, Ravishankar S, Bright KR. Antimicrobial efficacy of plant essential oils and extracts against Escherichia coli. J Environ Sci Health A Tox Hazard Subst Environ Eng. (2019) 54:608-16. doi: 10.1080/10934529.2019.1574153

23. Almayouf MA, El-Khadragy MF, Awad MA, Al-Olayan EM. The effects of silver nanoparticles biosynthesized using fig and olive extracts on cutaneous leishmaniasis induced inflammation in female Balb/c Mice. Biosci Rep. (2020) 40:BSR20202672. doi: 10.1042/BSR20202672

24. Basiricò L, Morera P, Dipasquale D, Bernini R, Santi L, Romani A, et al. (-)-Epigallocatechin-3-gallate and hydroxytyrosol improved antioxidative and anti-inflammatory responses in bovine mammary epithelial cells. Animal. (2019) 13:2847-56. doi: 10.1017/s1751731119001356

25. Yonezawa Y, Miyashita T, Nejishima H, Takeda Y, Imai K, Ogawa H. Antiinflammatory effects of olive-derived hydroxytyrosol on lipopolysaccharideinduced inflammation in RAW264.7 cells. J Vet Med Sci. (2018) 80:18017. doi: 10.1292/jvms.18-0250

26. Fonollá J, Maldonado-Lobón JA, Luque R, Rodríguez C, Bañuelos Ó, López-Larramendi JL, et al. Effects of a combination of extracts from olive fruit and almonds skin on oxidative and inflammation markers in hypercholesterolemic subjects: a randomized controlled trial. J Med Food. (2020). doi: 10.1089/jmf.2020.0088. [Epub ahead of print].

27. Scoditti E, Carpi S, Massaro M, Pellegrino M, Polini B, Carluccio MA, et al. Hydroxytyrosol modulates adipocyte gene and miRNA expression under inflammatory condition. Nutrients. (2019) 11:2493. doi: 10.3390/nu11102493

28. Cardona F, Andrés-Lacueva C, Tulipani S, Tinahones FJ, Queipo-Ortuño MI. Benefits of polyphenols on gut microbiota and implications in human health. J Nutr Biochem. (2013) 24:1415-22. doi: 10.1016/j.jnutbio.2013.05.001

29. Santhakumar AB, Battino M, Alvarez-Suarez JM. Dietary polyphenols: structures, bioavailability and protective effects against atherosclerosis. Food Chem Toxicol. (2018) 113:49-65. doi: 10.1016/j.fct.2018.01.022

30. Conterno L, Martinelli F, Tamburini M, Fava F, Mancini A, Sordo M, et al. Measuring the impact of olive pomace enriched biscuits on the gut microbiota and its metabolic activity in mildly hypercholesterolaemic subjects. Eur J Nutr. (2019) 58:63-81. doi: 10.1007/s00394-017-1572-2

31. Millman J, Okamoto S, Kimura A, Uema T, Higa M, Yonamine M, et al. Metabolically and immunologically beneficial impact of extra virgin olive and flaxseed oils on composition of gut microbiota in mice. Eur J Nutr. (2020) 59:2411-25. doi: 10.1007/s00394-019-02088-0

32. Vezza T, Rodríguez-Nogales A, Algieri F, Garrido-Mesa J, Romero M, Sánchez $\mathrm{M}$, et al. The metabolic and vascular protective effects of olive (Olea europaea L.) leaf extract in diet-induced obesity in mice are related to the amelioration of gut microbiota dysbiosis and to its immunomodulatory properties. Pharmacol Res. (2019) 150:104487. doi: 10.1016/j.phrs.2019.104487

33. Kahroba H, Ramezani B, Maadi H, Sadeghi MR, Jaberie H, Ramezani F. The role of $\mathrm{Nrf2}$ in neural stem/progenitors cells: from maintaining stemness and self-renewal to promoting differentiation capability and facilitating therapeutic application in neurodegenerative disease. Ageing Res Rev. (2020) 65:101211. doi: 10.1016/j.arr.2020.101211

34. Stern M, McNew JA. A transition to degeneration triggered by oxidative stress in degenerative disorders. Mol Psychiatry. (2021) 26:736-46. doi: 10.1038/s41380-020-00943-9

35. Vatner SF, Zhang J, Oydanich M, Berkman T, Naftalovich R, Vatner DE. Healthful aging mediated by inhibition of oxidative stress. Ageing Res Rev. (2020) 64:101194. doi: 10.1016/j.arr.2020.101194

36. Martín-Vertedor D, Garrido M, Pariente JA, Espino J, Delgado-Adámez J. Bioavailability of bioactive molecules from olive leaf extracts and its functional value. Phytother Res. (2016) 30:1172-9. doi: 10.1002/ptr.5625

37. Medina E, Romero C, García P, Brenes M. Characterization of bioactive compounds in commercial olive leaf extracts, and olive leaves and their infusions. Food Funct. (2019) 10:4716-24. doi: 10.1039/c9fo00698b

38. Gorzynik-Debicka M, Przychodzen P, Cappello F, Kuban-Jankowska A, Marino Gammazza A, Knap N, et al. Potential health benefits of olive oil and plant polyphenols. Int J Mol Sci. (2018) 19:686. doi: 10.3390/ijms19030686

39. Martínez L, Castillo J, Ros G, Nieto G. Antioxidant and antimicrobial activity of rosemary, pomegranate and olive extracts in fish patties. Antioxidants (Basel). (2019) 8:86. doi: 10.3390/antiox 8040086 
40. Schaffer S, Podstawa M, Visioli F, Bogani P, Müller WE, Eckert GP. Hydroxytyrosol-rich olive mill wastewater extract protects brain cells in vitro and ex vivo. J Agric Food Chem. (2007) 55:5043-9. doi: 10.1021/jf0703710

41. Sun L, Luo C, Liu J. Hydroxytyrosol induces apoptosis in human colon cancer cells through ROS generation. Food Funct. (2014) 5:190914. doi: 10.1039/c4fo00187g

42. Crupi R, Palma E, Siracusa R, Fusco R, Gugliandolo E, Cordaro M, et al. Protective effect of hydroxytyrosol against oxidative stress induced by the ochratoxin in kidney cells: in vitro and in vivo Study. Front Vet Sci. (2020) 7:136. doi: 10.3389/fvets.2020.00136

43. Loru D, Incani A, Deiana M, Corona G, Atzeri A, Melis MP, et al. Protective effect of hydroxytyrosol and tyrosol against oxidative stress in kidney cells. Toxicol Ind Health. (2009) 25:301-10. doi: 10.1177/0748233709103028

44. Martínez-Lara E, Peña A, Calahorra J, Cañuelo A, Siles E. Hydroxytyrosol decreases the oxidative and nitrosative stress levels and promotes angiogenesis through HIF-1 independent mechanisms in renal hypoxic cells. Food Funct. (2016) 7:540-8. doi: 10.1039/c5fo00928f

45. Lei XG, Zhu JH, Cheng WH, Bao Y, Ho YS, Redd2i AR, et al. Paradoxical roles of antioxidant enzymes: basic mechanisms and health implications. Physiol Rev. (2016) 96:307-64. doi: 10.1152/physrev.00010.2014

46. He L, He T, Farrar S, Ji L, Liu T, Ma X. Antioxidants maintain cellular redox homeostasis by elimination of reactive oxygen species. Cell Physiol Biochem. (2017) 44:532-53. doi: 10.1159/000485089

47. Chen M, Cai W, Zhao S, Shi L, Chen Y, Li X, et al. Oxidative stress-related biomarkers in saliva and gingival crevicular fluid associated with chronic periodontitis: a systematic review and meta-analysis. J Clin Periodontol. (2019) 46:608-22. doi: 10.1111/jcpe.13112

48. Ahlawat S, Asha, Sharma KK. Gut-organ axis: a microbial outreach and networking. Lett Appl Microbiol. (2020). doi: 10.1111/lam.13333. [Epub ahead of print].

49. Ringseis R, Gessner DK, Eder K. The gut-liver axis in the control of energy metabolism and food intake in animals. Annu Rev Anim Biosci. (2020) 8:295-319. doi: 10.1146/annurev-animal-021419-083852

50. Fraga CG, Croft KD, Kennedy DO, Tomás-Barberán FA. The effects of polyphenols and other bioactives on human health. Food Funct. (2019) 10:514-28. doi: 10.1039/c8fo01997e

51. Gessner DK, Ringseis R, Eder K. Potential of plant polyphenols to combat oxidative stress and inflammatory processes in farm animals. J Anim Physiol Anim Nutr (Berl). (2017) 101:605-28. doi: 10.1111/jpn.12579

52. Zhang $\mathrm{H}$, Davies KJA, Forman HJ. Oxidative stress response and Nrf2 signaling in aging. Free Radic Biol Med. (2015) 88:314-36. doi: 10.1016/j.freeradbiomed.2015.05.036

53. Marinić J, Broznić D, Milin C. Preexposure to olive oil polyphenols extract increases oxidative load and improves liver mass restoration after hepatectomy in mice via stress-sensitive genes. Oxid Med Cell Longev. (2016) 2016:9191407. doi: 10.1155/2016/9191407

54. Has AL, Alotaibi MF, Bin-Jumah M, Elgebaly H, Mahmoud AM. Olea europaea leaf extract up-regulates Nrf2/ARE/HO-1 signaling and attenuates cyclophosphamide-induced oxidative stress, inflammation and apoptosis in rat kidney. Biomed Pharmacother. (2019) 111:676-85. doi: 10.1016/j.biopha.2018.12.112

55. Omidian K, Rafiei H, Bandy B. Polyphenol inhibition of benzo[a]pyreneinduced oxidative stress and neoplastic transformation in an in vitro model of carcinogenesis. Food Chem Toxicol. (2017) 106:165-74. doi: 10.1016/j.fct.2017.05.037

56. Yahfoufi N, Alsadi N, Jambi M, Matar C. The immunomodulatory and anti-inflammatory role of polyphenols. Nutrients. (2018) 10:1618. doi: 10.3390/nu10111618

57. Wauquier F, Mevel E, Krisa S, Richard T, Valls J, Hornedo-Ortega R, et al. Chondroprotective properties of human-enriched serum following polyphenol extract absorption: results from an exploratory clinical trial. Nutrients. (2019) 11:9071. doi: 10.3390/nu11123071

58. Kountouri AM, Kaliora AC, Koumbi L, Andrikopoulos NK. In-vitro gastric cancer prevention by a polyphenol-rich extract from olives through induction of apoptosis. Eur J Cancer Prev. (2009) 18:339. doi: 10.1097/CEJ.0b013e3282fb75f7

59. Tuck KL, Freeman MP, Hayball PJ, Stretch GL, Stupans I. The in vivo fate of hydroxytyrosol and tyrosol, antioxidant phenolic constituents of olive oil, after intravenous and oral dosing of labeled compounds to rats. J Nutr. (2001) 131:1993-6. doi: 10.1093/jn/131.7.1993

60. Visioli F, Galli C, Bornet F, Mattei A, Patelli R, Galli G, et al. Olive oil phenolics are dose-dependently absorbed in humans. FEBS Lett. (2000) 468:159-60. doi: 10.1016/s0014-5793(00)01216-3

61. D'Angelo S, Manna C, Migliardi V, Mazzoni O, Morrica P, Capasso G, et al. Pharmacokinetics and metabolism of hydroxytyrosol, a natural antioxidant from olive oil. Drug Metab Dispos. (2001) 29:1492-8. doi: 10.1016/S1359-6446(01)01977-8

62. Gaupp R, Ledala N, Somerville GA. Staphylococcal response to oxidative stress. Front Cell Infect Microbiol. (2012) 2:33. doi: 10.3389/fcimb.2012.00033

63. Giuliani C, Marzorati M, Daghio M, Franzetti A, Innocenti M, Van de Wiele $\mathrm{T}$, et al. Effects of olive and pomegranate by-products on human microbiota: a study using the SHIME $\left({ }^{\circledR}\right)$ in vitro simulator. Molecules. (2019) 24:3791. doi: 10.3390/molecules24203791

64. Wang $\mathrm{H}, \mathrm{Hu} \mathrm{C}$, Cheng $\mathrm{C}$, Cui J, Ji Y, Hao X, et al. Unraveling the association of fecal microbiota and oxidative stress with stillbirth rate of sows. Theriogenology. (2019) 136:131-7. doi: 10.1016/j.theriogenology.2019.06.028

65. Qiao Y, Sun J, Ding Y, Le G, Shi Y. Alterations of the gut microbiota in highfat diet mice is strongly linked to oxidative stress. Appl Microbiol Biotechnol. (2013) 97:1689-97. doi: 10.1007/s00253-012-4323-6

66. Li Y, Liu H, Zhang L, Yang Y, Lin Y, Zhuo Y, et al. Maternal dietary fiber composition during gestation induces changes in offspring antioxidative capacity, inflammatory response, and gut microbiota in a sow model. Int J Mol Sci. (2019) 21:31. doi: 10.3390/ijms21010031

67. Nie Y, Hu J, Hou Q, Zheng W, Zhang X, Yang T, et al. Lactobacillus frumenti improves antioxidant capacity via nitric oxide synthase 1 in intestinal epithelial cells. Faseb J. (2019) 33:10705-16. doi: 10.1096/fj.201900253RR

68. Louis P, Flint HJ. Formation of propionate and butyrate by the human colonic microbiota. Environ Microbiol. (2017) 19:29-41. doi: 10.1111/1462-2920.13589

69. Ortega-Hernández A, Martínez-Martínez E, Gómez-Gordo R, López-Andrés N, Fernández-Celis A, Gutiérrrez-Miranda B, et al. The interaction between mitochondrial oxidative stress and gut microbiota in the cardiometabolic consequences in diet-induced obese rats. Antioxidants (Basel). (2020) 9:640. doi: 10.3390/antiox9070640

70. Mariat D, Firmesse O, Levenez F, Guimarăes V, Sokol H, Doré J, et al. The Firmicutes/Bacteroidetes ratio of the human microbiota changes with age. BMC Microbiol. (2009) 9:123. doi: 10.1186/1471-2180-9-123

71. Chae JP, Pajarillo EA, Oh JK, Kim H, Kang DK. Revealing the combined effects of lactulose and probiotic enterococci on the swine faecal microbiota using 454 pyrosequencing. Microb Biotechnol. (2016) 9:48695. doi: 10.1111/1751-7915.12370

72. Molist F, Manzanilla EG, Pérez JF, Nyachoti CM. Coarse, but not finely ground, dietary fibre increases intestinal Firmicutes:Bacteroidetes ratio and reduces diarrhoea induced by experimental infection in piglets. Br J Nutr. (2012) 108:9-15. doi: 10.1017/s0007114511005216

73. Hu R, He Z, Liu M, Tan J, Zhang H, Hou DX, et al. Dietary protocatechuic acid ameliorates inflammation and up-regulates intestinal tight junction proteins by modulating gut microbiota in LPS-challenged piglets. J Anim Sci Biotechnol. (2020) 11:92. doi: 10.1186/s40104-020-0 0492-9

74. Yu Z, Zhao-Xi D, Mao-Long H, Xin ML, Jian-Xin L, Haifeng W. Olive extract ameliorates oxidative stress and inflammation, and protects intestinal villus and microbiota in piglets induced by Lipopolysaccharides. Res Square. (2021). doi: 10.21203/rs.3.rs-101171/v1. [Epub ahead of print].

Conflict of Interest: The authors declare that the research was conducted in the absence of any commercial or financial relationships that could be construed as a potential conflict of interest.

Copyright (c) 2021 Wang, Zhang, Zhong, Wan, Chen, Liu, Yi and Zhang. This is an open-access article distributed under the terms of the Creative Commons Attribution License (CC BY). The use, distribution or reproduction in other forums is permitted, provided the original author(s) and the copyright owner(s) are credited and that the original publication in this journal is cited, in accordance with accepted academic practice. No use, distribution or reproduction is permitted which does not comply with these terms. 